\title{
Burden and risk factors of invasive group $B$ Streptococcus disease among neonates in a Chinese maternity hospital
}

\author{
Qunhua Ying ${ }^{1 *}$, Shutan Wang ${ }^{2}$, Xiuming Lou ${ }^{1}$, Jinlong Ding ${ }^{1}$ and Jiefeng Ding ${ }^{2}$
}

\begin{abstract}
Background: There is a lack of data regarding the prevalence of invasive group B streptococcus (GBS) infection among neonates in China. This study aimed to investigate the incidence and mortality of invasive GBS infection and to identify the risk factors in our hospital.

Methods: Seventy-four cases admitted between January 2011 and December 2016 was included in this study. A retrospective matched case-control study was conducted in a tertiary maternity and paediatric hospital. Risk factors for the acquisition of invasive GBS infection and mortality were analysed by univariable and multivariable analysis.

Results: We collected and analysed data from 74 infants aged $<3$ months with invasive GBS infection. Among 67,985 live births, we calculated an incidence of 1.09 per 1000 live births (95\%Cl:0.81-1.37\%); the incidence of Earlyonset GBS disease (EOD, $n=65$ ) and Late-onset GBS disease (LOD, $n=9$ ) were 0.96\%o(95\%Cl:0.73-1.19\%) and 0. 13\%o(95\%Cl:0.04-0.22\%), respectively. Overall, pneumonia accounted $63.1 \%(41 / 65)$ of EOD, and sepsis accounted $88.9 \%$ (8/9) cases of LOD, respectively. The overall case fatality rate was $8.11 \%(6 / 74)$, including $7.69 \%(5 / 65)$ among cases of EOD and $11.1 \%(1 / 9)$ among cases of LOD. No predictor of mortality was found. Membrane stripping $(P=$ 0.005, aOR: 3.68, 95\% Cl: 1.48-9.13) and non-resident mother ( $P<0.001$, aOR: 5.88, 95\% Cl: 2.36-14.61) were independent risk factors for EOD; no increased risk was found for LOD.

Conclusions: This study demonstrates remarkable country-specific variation in comparison with other countries. Our findings can improve awareness of neonatal GBS infection and lay a cornerstone to ensure accurate representation of the burden.
\end{abstract}

Keywords: Streptococcus group B (GBS), Neonatal Sepsis, GBS risk factors, Early-onset disease (EOD), Late-onset disease (LOD)

\section{Background}

Group B Streptococcus (GBS) is a leading cause of invasive neonatal infection [1]. There are several previous studies of the burden and risk factors for invasive GBS disease in many countries [2-4]. However, there is a lack of data on the prevalence of invasive GBS infection among neonates in China. China's new two-child policy will result in more infants being born each year; therefore, reducing neonatal GBS infection is important. Hence, we performed a retrospective study to evaluate the risk factors

\footnotetext{
* Correspondence: ssbx1972@163.com

'Department of Clinical Laboratory, Shaoxing Women and Children's

Hospital, 305 East Street Road, Shaoxing 312000, Zhejiang, China

Full list of author information is available at the end of the article
}

and burden for invasive neonatal GBS infection in our hospital in east China.

\section{Methods \\ Study setting}

We undertook a retrospective case-control study to evaluate the incidence, mortality, risk factors, and characteristics associated with invasive neonatal GBS infection. Cases were treated at Shaoxing Maternity and Child Care Hospital, a 600-bed modern hospital specializing in Gynaecology, Obstetrics and Paediatrics in Shaoxing, Zhejiang Province in east China. Approximately 10,000 infants are delivered each year, and GBS screening and intrapartum antibiotic prophylaxis (IAP)

(c) The Author(s). 2019 Open Access This article is distributed under the terms of the Creative Commons Attribution 4.0 International License (http://creativecommons.org/licenses/by/4.0/), which permits unrestricted use, distribution, and 
during pregnancy is not standard of care. Risk factors were assessed by comparing infants with invasive neonatal GBS infection to those without, and variables assessed as possible risk factors for invasive GBS disease included previously identified risk factors, such as preterm delivery ( $<37$ weeks' gestational age), prolonged rupture of membranes $(>=18 \mathrm{~h}$ ), birth weight $<2500 \mathrm{~g}$, known genital GBS colonization, intrapartum fever, and non-use of IAP. Resident status (mothers whether or not being resident of Shaoxing) and membrane stripping (women who did and did not undergo membrane stripping) were also assessed.

\section{Study design and population}

Cases of GBS infection were defined as infants $<90$ days of age in whom GBS was isolated from blood or cerebrospinal fluid (CSF) and otherwise sterile body fluids. Investigation was performed from January 1, 2011 through December 31, 2016.Early onset disease (EOD) was defined when GBS was isolated in infants younger than 7 days of life, and infants 7-89 days of age with GBS disease were regarded as having late-onset disease (LOD) [5]. Cases were identified by medical records investigation of the paediatric wards and microbiology services. For each neonate with invasive GBS infection, we randomly selected two controls for cases of EOD and 3 controls for cases of LOD from infants who were born within the same period and had no bacterial infection.

\section{Microbiological identification and susceptibility testing}

Blood and throat swab cultures are routinely performed in infants admitted to the neonatal intensive care unit (NICU) with suspected sepsis or pneumonia. Cerebrospinal fluid (CSF) and other specimen cultures are performed in infants with suspected meningitis or other clinical manifestations. GBS was isolated from blood samples using the BACT/ALERT 3D microbial detection system (bio-Mérieux, Marcy-l'Étoile, France). Positive blood culture bottles were subsequently sub cultured to Columbia blood agar (bio-Mérieux, Marcy-l'Étoile, France) incubated aerobically at $35^{\circ} \mathrm{C}$ under $5-10 \% \mathrm{CO}_{2}$ and observed for colony growth for $48 \mathrm{~h}$. CSF samples were inoculated into 3D blood culture bottles and immediate Gram stain was performed. Other swabs such as eye swab and umbilical exudation swab were plated onto Columbia blood agar plates that were incubated at $35^{\circ} \mathrm{C}$ for $18-24 \mathrm{~h}$ under $5-10 \% \mathrm{CO}_{2}$ and examined for growth of $ß$-haemolytic GBS-like colony morphology. Presumptive identification of GBS was based on Gram stain and a positive CAMP test, determined by an arrowhead-shaped zone of complete haemolysis. Vitek 2 COMPACT (bio-Mérieux, Marcy-l'Étoile, France) was used to perform identification and susceptibility testing.

\section{Data collection}

Data were obtained from medical records, and relevant data were recorded on questionnaires. During the study period the one-child policy was in place and as such may effect risk calculation.Only 2 mothers of cases had previous children, each of whom died due to pneumonia of unknown pathogen.

\section{Statistical analysis}

Continuous variables were compared with the Student t-test (for normally distributed variables) or the MannWhitney $\mathrm{U}$ test (for non-normally distributed variables) and are presented as the mean \pm standard deviation (SD) or median (range). Categorical variables are presented as percentages and evaluated using the chi-square $\left(x^{2}\right)$ test or the two-tailed Fischer's exact test. Univariable analysis and multivariable analysis were used to identify risk factors for invasive GBS disease and mortality. We performed univariable analyses to compare the cases and controls in terms of risk factors. The association between independent variables is shown as the odds ratio with 95\% confidence intervals, and variables for which the $P$ value was less than 0.10 in the univariable analysis were included in a conditional logistic regression model for multivariable analysis. For the multivariable analysis, adjusted odds ratios (aOR) using conditional logistic regression were used to adjust for variables deemed significant by univariable analysis. The odds ratio (OR),the confidence interval (CI) and $P$-value are used to figure out if a exposure is a risk factor for EOD and LOD, and to compare the various risk factors for GBS-disease happening. An OR of greater than 1 means that there is a higher odds of disease happening with exposure to a risk factor. An OR is less than 1 is associated with lower odds. An OR of exactly 1 implies that exposure to factor does not affect the odds of disease. A two-tailed $P$ value of less than 0.05 was considered to show statistical significance, and statistical analyses were performed using SPSS 19.0 (SPSS, Inc., Chicago, IL, USA).

\section{Results \\ Incidence and risk factors of invasive neonatal GBS infection}

The incidence of invasive neonatal GBS infection over the 6-year study period is presented in Fig. 1.During the 6 -year study period, there were 74 infants $(<90$ days of age) with invasive GBS disease among 67,985 live births (LBs),yielding an overall incidence (per $1000 \mathrm{LBs}$ ) of invasive GBS disease of $1.09 \%$ (95\%CI:0.81\%-1.37\%); the incidences of $\operatorname{EOD}(n=65)$ and LOD $(n=9)$ were 0.96\%o(95\%CI:0.73\%o-1.19\%o) and 0.13\%o(95\%CI:0.04\%o$0.22 \%$ ), respectively. There were 9 cases among 10,117 LBs in 2011(0.89/1000 LBs), 5 cases among 10,855 L Bs in2012 (0.46/1000 LBs), 10 cases among 10,463 LBs in 


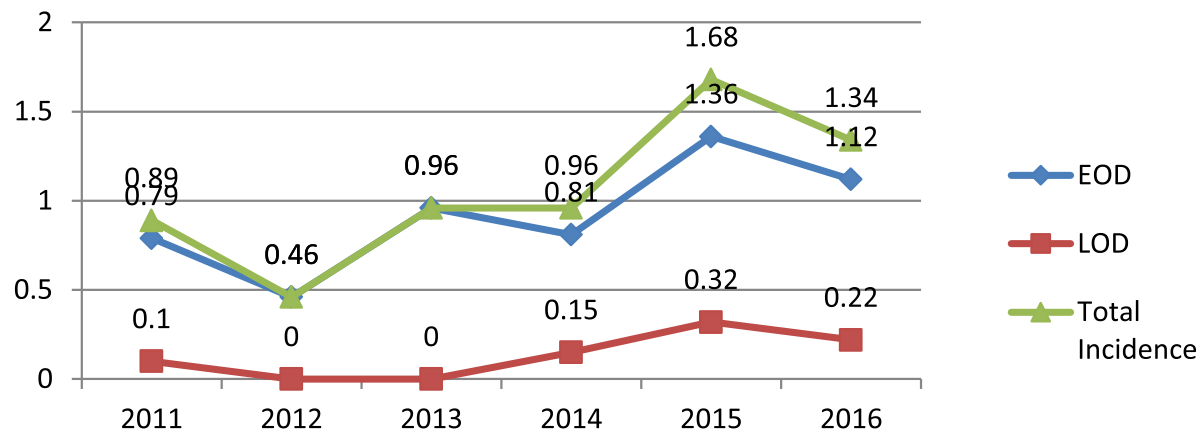

Fig 1. The incidence of invasive neonatal GBS infection over the 6-years

2013(0.96/1000 LBs), 13 cases among 13,569 LBs in 2014 (0.96/1000 LBs), 16 cases among 9552 LBs in 2015(1.68/1000 LBs), and 18 cases among 13,429 LBs in 2016(1.34/1000 LBs).

Risk factors for invasive Group B Streptococcal (GBS) disease in EOD and LOD cases and matched controls are presented in Table 1 . In total 28/65 (43.1\%) EOD cases occurred in either non-residents of the city or patients undergoing membrane stripping.Membrane stripping ( $P=$ 0.005, aOR: 3.68, 95\% CI: 1.48-9.13) and non-resident mother $(P<0.001$,aOR: 5.88, 95\% CI: 2.36-14.61)were associated with a greater risk for EOD in the univariable analysis, whereas previously described risk factors such as preterm delivery ( $<37$ weeks' gestational age), prolonged rupture of membranes ( $>=18 \mathrm{~h}$ ), birth weight $<2500 \mathrm{~g}$, known genital GBS colonization, intrapartum fever, and non-use of IAP. According to ORs ordered by their magnitude, 5 increased risk factors found in the multivariable analysis $(P<0.05)$ ranked as follows: intrapartum temperature $>=38.0^{\circ} \mathrm{C}$, GBS screened positive, non-resident mother, rupture of membranes for > $=18 \mathrm{~h}$, membrane stripping.

All 9 cases with LOD were born to women with negative vaginal swab cultures. Although the eight identified factors were more common in cases with LOD than in controls, no increased risk was found in the chi-square analysis $(P>0.05)$.

\section{Clinical data on EOD and LOD cases}

Comparisons between the demographic characteristics of the EOD and LOD groups are shown in Table 2. No differences were found in demographic characteristics

Table 1 Risk factors for invasive Group B Streptococcal (GBS) disease in EOD and LOD cases and matched controls

\begin{tabular}{|c|c|c|c|c|c|c|}
\hline & Cases & Controls & Univariable-OR(95\%Cl) & $P$-value & Multivariable-OR(95\%Cl) & $P$-value \\
\hline EOD & $(n=65)$ & $(n=130)$ & & & & \\
\hline Non-resident mother & 28 & 20 & $4.16(2.10-8.25)$ & $<0.001$ & $5.88(2.36-14.61)$ & $<0.001$ \\
\hline GBS screened positive & 17 & 5 & $8.85(3.10-25.33)$ & $<0.001$ & 10.97(3.02-39.88) & $<0.001$ \\
\hline Intrapartum temperature $>=38.0^{\circ} \mathrm{C}$ & 22 & 2 & $32.74(7.39-145.03)$ & $<0.001$ & $30.78(5.37-176.53)$ & $<0.001$ \\
\hline Membrane stripping & 28 & 30 & $2.52(1.33-4.78)$ & 0.004 & $3.68(1.48-9.13)$ & 0.005 \\
\hline Rupture of membranes for $>=18 \mathrm{~h}$ & 22 & 14 & 4.24(1.99-9.03) & $<0.001$ & $4.16(1.35-12.80)$ & 0.013 \\
\hline No IAP & 29 & 106 & $0.18(0.09-0.35)$ & $<0.001$ & $2.14(0.82-5.59)$ & 0.122 \\
\hline Gestational Age $<37$ weeks & 17 & 13 & $3.19(1.44-7.07)$ & 0.003 & $1.29(0.35-4.68)$ & 0.701 \\
\hline Birth Weight < $2500 \mathrm{~g}$ & 14 & 8 & $4.19(1.66-10.60)$ & 0.001 & 2.82(0.70-11.41) & 0.147 \\
\hline LOD & $(n=9)$ & $(n=27)$ & & & & \\
\hline Non-resident mother & 4 & 4 & $4.60(0.85-24.93)$ & 0.086 & & \\
\hline GBS screened positive & 0 & 1 & & 1.000 & & \\
\hline Intrapartum temperature $>=38.0^{\circ} \mathrm{C}$ & 1 & 0 & & 0.250 & & \\
\hline Membrane stripping & 5 & 7 & $3.57(0.74-17.20)$ & 0.126 & & \\
\hline Rupture of membranes for $>=18 \mathrm{~h}$ & 1 & 1 & $3.25(0.18-58.06)$ & 0.443 & & \\
\hline No IAP & 7 & 26 & $0.14(0.01-1.71)$ & 0.148 & & \\
\hline Gestational Age $<37$ weeks & 2 & 2 & $3.57(0.42-30.10)$ & 0.255 & & \\
\hline Birth Weight < $2500 \mathrm{~g}$ & 2 & 3 & $2.29(0.32-16.51)$ & 0.581 & & \\
\hline
\end{tabular}


Table 2 Demographic characteristics of infants with invasive Group B Streptococcal (GBS) disease

\begin{tabular}{|c|c|c|c|c|c|}
\hline Varibles & $\begin{array}{l}\text { All cases } \\
(n=74)\end{array}$ & $\begin{array}{l}\mathrm{EOD}^{1} \\
(n=65)\end{array}$ & $\begin{array}{l}\mathrm{LOD}^{2} \\
(n=9)\end{array}$ & $\mathrm{OR}(95 \% \mathrm{Cl})^{3}$ & $p$-value ${ }^{4}$ \\
\hline \multicolumn{6}{|l|}{ Gender } \\
\hline Male & 33 & $27(41.5)$ & $6(66.7)$ & $0.36(0.08-1.55)$ & 0.176 \\
\hline \multicolumn{6}{|l|}{ Gestational Age } \\
\hline$\geq 37$ weeks & 55 & 48(73.8) & $7(77.8)$ & $0.81(0.15-4.27)$ & 1 \\
\hline$<37$ weeks & 19 & $17(26.2)$ & $2(22.2)$ & $1.24(0.23-6.56)$ & 1 \\
\hline$<37-\geq 34$ weeks & 13 & $12(18.5)$ & $1(11.1)$ & $1.81(0.21-15.89)$ & 1 \\
\hline$<34$ weeks & 6 & $5(7.7)$ & $1(11.1)$ & $0.67(0.07-6.45)$ & 0.554 \\
\hline \multicolumn{6}{|l|}{ Birth Weight } \\
\hline$\geq 2500 \mathrm{~g}$ & 58 & $51(78.5)$ & $7(77.8)$ & $1.04(0.19-5.58)$ & 1 \\
\hline$<2500 \mathrm{~g}$ & 16 & $14(21.5)$ & $2(22.2)$ & $0.96(0.18-5.15)$ & 1 \\
\hline $1500-2499 \mathrm{~g}$ & 15 & $13(20)$ & 22(22.2) & $0.88(0.16-4.72)$ & 1 \\
\hline$<1500 \mathrm{~g}$ & 1 & $1(1.5)$ & $0(0)$ & & 1 \\
\hline \multicolumn{6}{|l|}{ Mode of delivery 1} \\
\hline Vertex delivery & 42 & $35(53.8)$ & $7(77.8)$ & $0.33(0.06-1.73)$ & 0.284 \\
\hline Caesarean-section & 28 & $26(40)$ & $2(22.2)$ & $2.33(0.45-12.13)$ & 0.468 \\
\hline Obstetric forceps & 4 & $4(6.2)$ & $0(0)$ & & 1 \\
\hline Membrane sweeping & 33 & $28(43.1)$ & $5(55.6)$ & $0.61(0.15-2.46)$ & 0.501 \\
\hline Non-resident mother & 32 & $28(43.1)$ & $4(44.4)$ & $0.95(0.23-3.85)$ & 1 \\
\hline
\end{tabular}

${ }^{1}$ EOD-Early-onset disease

2 LOD-Late-onset disease

${ }^{3} \mathrm{OR}(95 \% \mathrm{Cl})$-calculated odds ratio with $95 \%$ confidence comparing EOD to LOD

${ }^{4} p$-value-using Chi-squared, Fischer exact or Wilcoxon rank-sum (Mann-Whitney) test

such as gestational age, birth weight or mode of delivery between infants with EOD and those with LOD.

Pneumonia with or without sepsis accounted 63.1\% $(41 / 65)$ of EOD. For infants with EOD, GBS was isolated from sputum in $34 / 65(52.3 \%)$, blood in $18 / 65(27.7 \%)$, blood and sputum in 7/65 (10.8\%), blood and CSF in 1/ $15(1.5 \%)$, and other samples (eye, urine, umbilicus) in $6 / 65(9.2 \%)$. Jaundice, asphyxia, fever, or preterm delivery were the initial symptoms for $8 / 65(12.3 \%), 5 / 65(7 / 7 \%)$, 6/65(9.2\%), and 12/65(18.5\%) infants with EOD, respectively. Respiratory symptoms including pneumonia at presentation $(43 / 65,66.2 \%, P=0.024)$ or mild illness (urethritis, omphalitis, and ophthalmia) were more common among infants with EOD than among infants with LOD.

In contrast, fever at presentation $(6 / 9,66.7 \%, P<$ $0.001)$ was significantly more frequent among infants with LOD. Sepsis accounted 88.9\% (8/9) cases of LOD. Additionally, the incidence of sepsis, shock, and meningitis was higher, though not significantly among infants with LOD. For 8/9 infants with LOD, GBS was isolated from blood, and it was isolated from sputum in 1/9.

$53 / 65(81.5 \%)$ infants with EOD were admitted to the NICU $<1$ day after birth. 8 / 9 infants with LOD were identified at $<30$ days. The median (range) infant age at presentation of EOD and LOD was 23 min (5 min-6 days) and 17 days $(7-51$ days), respectively $(P<0.001)$. The average length of hospitalization in NICU of EOD and LOD cases was 9.37 and 10.67 days $(P=0.046)$.

Obstetric characteristics of women whose infants developed EOD stratified by delivery method are shown in Table 3. There was no significant difference in the GBS infection rates by the three delivery modes. Among 26/ 74 (35.1\%) women who underwent caesarean delivery and whose infant developed EOD, 25/26 (96.2\%) were delivered after rupture of membranes, and the remaining woman was GBS-positive. Caesarean section was performed in response to dystocia, and 21/26 of women undergoing $\mathrm{C}$-section received antibiotics. Most infants $(56 / 74,75.7 \%)$ were born to women with $>1$ risk factor. 38/74(51.4\%) women received IAP with different antibiotic dose and exposure duration before delivery. Erythromycin enteric capsule was commenced $12 \mathrm{~h}$ after rupture of membranes. Combined use of antibiotics can also be seen.

\section{Case fatality rate}

The overall case fatality rate among cases was $8.11 \%$ (6/ $74)$, including $7.69 \%(5 / 65)$ for infants with EOD and $11.1 \%(1 / 9)$ for infants with LOD. While the difference in the mortality rate between infants with EOD and those with LOD seems large, it is not statistically 
Table 3 Obstetric characteristics of women whose infants developed EOD and LOD stratified by delivery method

\begin{tabular}{|c|c|c|c|c|c|c|c|c|c|c|c|c|c|}
\hline \multirow[t]{2}{*}{$\begin{array}{l}\text { Mode of } \\
\text { delivery }\end{array}$} & \multirow[t]{2}{*}{$\begin{array}{l}\text { Membrane } \\
\text { sweeping }\end{array}$} & \multirow{2}{*}{$\begin{array}{l}\text { Intrapartum } \\
\text { temperature } \\
\geq 38^{\circ} \mathrm{C}\end{array}$} & \multicolumn{2}{|c|}{$\begin{array}{l}\text { Rupture of } \\
\text { membranes }\end{array}$} & \multirow{2}{*}{$\begin{array}{l}\text { Urine } \\
\text { WBC } \\
+\end{array}$} & \multirow[t]{2}{*}{ Asphyxia } & \multicolumn{7}{|c|}{ Intrapartum Antibiotic Prophylaxis } \\
\hline & & & $\begin{array}{l}>= \\
18 \mathrm{~h}\end{array}$ & $\begin{array}{l}< \\
18 \mathrm{~h}\end{array}$ & & & No & erythromycin & cefazolin & cefuroxime & ampicillin & penicillin & others \\
\hline \multicolumn{14}{|l|}{ EOD } \\
\hline $\begin{array}{l}\text { Obstetric } \\
\text { forceps } \\
(n=4)\end{array}$ & 3 & 1 & 1 & 1 & 0 & 3 & 2 & 0 & 1 & 0 & 1 & 0 & 0 \\
\hline $\begin{array}{l}\text { Caesarean- } \\
\text { section } \\
(n=26)\end{array}$ & 10 & 13 & 15 & 10 & 5 & 7 & 5 & 7 & 5 & 7 & 0 & 1 & 1 \\
\hline $\begin{array}{l}\text { Vaginal } \\
\text { delivery(35) }\end{array}$ & 15 & 8 & 6 & 12 & 6 & 9 & 22 & 5 & 4 & 1 & 0 & 3 & 1 \\
\hline \multicolumn{14}{|l|}{ LOD } \\
\hline $\begin{array}{l}\text { Obstetric } \\
\text { forceps } \\
(n=0)\end{array}$ & 0 & 0 & 0 & 0 & 0 & 0 & 0 & 0 & 0 & 0 & 0 & 0 & 0 \\
\hline $\begin{array}{l}\text { Caesarean- } \\
\text { section } \\
(n=2)\end{array}$ & 1 & 1 & 1 & 0 & 0 & 0 & 1 & 1 & 0 & 0 & 0 & 1 & 0 \\
\hline $\begin{array}{l}\text { Vaginal } \\
\text { delivery } \\
(n=7)\end{array}$ & 4 & 0 & 0 & 1 & 1 & 1 & 6 & 2 & 2 & 0 & 0 & 3 & 0 \\
\hline
\end{tabular}

significant $[P=0.55, \mathrm{OR}=0.67$ (95\%CI: 0.07-6.45)]. Possible predictors of mortality are shown in Table 4 but none were significant. The mothers in all 6 cases had low vaginal swabs collected at hospital admission were reported to be GBS-negative. Maternal urinary tract culture was seldom performed. Two mothers with positive urine white blood cell counts did not undergo urine culture. Only one case was GBS-positive based on CSF.GBS was detected in the blood cultures of all fatal cases with EOD. Treatment was discontinued in cases where the outcome was considered to be poor, which included a child with severe meningitis, intracranial haemorrhage, collodion baby, and prematurity with respiratory failure.
The other infant died within half an hour after delivery due to asphyxia. The average length of hospitalization in NICU among fatal cases was 4.83 days, which was less than the average of 9.94 days among $\operatorname{cured} \operatorname{cases}(P=$ 0.010). The mean birth weight among fatal and cured cases was 2.97 and $3.07 \mathrm{~kg}(P=0.707)$, and the mean gestational age was 38.7 and 37.8 weeks $(P=0.42)$, respectively.

\section{Discussion}

Over the past decades, GBS has been recognized as a main cause of neonatal infections associated with high morbidity and mortality [2, 5]. Striking declines in

Table 4 Predictors of deaths for neonatal GBS infections

\begin{tabular}{|c|c|c|c|c|}
\hline \multirow[t]{2}{*}{ Variables } & \multicolumn{2}{|c|}{ No.(\%)of patients } & \multirow[t]{2}{*}{ OR(95\%Cl) } & \multirow{2}{*}{$\begin{array}{l}P_{-} \\
\text {value }\end{array}$} \\
\hline & dead $(n=6)$ & survival $(n=68)$ & & \\
\hline Non-resident mother & $5(83.3)$ & $36(52.9)$ & $4.44(0.49-40.08)$ & 0.216 \\
\hline Asphyxia & $3(50)$ & $17(25)$ & $3.00(0.55-16.29)$ & 0.334 \\
\hline Tracheal intubation & $2(33.3)$ & 13(19.1) & $2.12(0.35-12.82)$ & 0.595 \\
\hline Membrane stripping & $2(33.3)$ & $31(45.6)$ & $0.60(0.10-3.48)$ & 0.686 \\
\hline Meningitis & $1(16.7)$ & $4(5.9)$ & $3.20(0.30-34.33)$ & 0.353 \\
\hline Sepsis & $5(83.3)$ & $27(39.7)$ & $7.59(0.84-68.61)$ & 0.079 \\
\hline Intrapartum Temperature $>=38.0^{\circ} \mathrm{C}$ & $3(50.0)$ & $20(29.4)$ & $2.40(0.45-12.92)$ & 0.367 \\
\hline Rupture of membranes for $>=18 \mathrm{~h}$ & $1(16.7)$ & $21(30.9)$ & $0.45(0.05-4.07)$ & 0.662 \\
\hline No IAP & $4(66.7)$ & $30(44.1)$ & $2.53(0.43-14.79)$ & 0.404 \\
\hline GBS screened positive & 0 & $32(47.1)$ & & 0.033 \\
\hline Central nervous system symptoms & $3(50.0)$ & 23(33.8) & $1.96(0.36-10.47)$ & 0.659 \\
\hline
\end{tabular}


disease incidence occurred following the issuance of guidelines from the $\mathrm{CDC}$ for prevention of perinatal group B streptococcal disease in 1996,2002, and 2010, most likely due to GBS screening and the implementation of IAP [2, 6-8]. There are no formal prevention policies in China. It is important to identify the disease characteristics to determine the best preventive strategy. Our data reveal local incidence, risk factors, clinical characteristics and case fatality rate that could serve as a baseline report for country-specific guidelines.

Since 2001, several studies have reported invasive neonatal GBS disease in China, with one paper reporting 118 infant cases across 7 hospitals between 2011 to 2015 [9-12]. However, there is a lack of systematic research [9-12]. Our study confirms that GBS is an important pathogen in fatal, non-fatal neonatal pneumonia, sepsis with or without pneumonia especially in EOD cases [9]. Retrospective chart review plotted the incidence of neonatal GBS infection as 1.1 per 1000 live births in Taiwan, and our study found a similar incidence [10].

Zhang reported that 5 (12.5\%) cases were classified as EOD and 35(87.5\%) were classified as LOD [11]. In the Netherlands, only $7 \%$ of all GBS infections are LOD, which contrasts with the United Kingdom and Germany, where 33.6 and $39.2 \%$ of cases are LOD, respectively [12].In our study, the incidence of EOD and LOD was $87.8 \%(65 / 74)$ and $12.2 \%(9 / 74)$, respectively. This difference may be associated with the differences in the patient population. Fewer cases of LOD in our study may be because infants aged $>1$ month may present to other local hospitals. Furthermore, receiving antibiotics at home or local community healthcare center (data is not collected) may lead to negative culture results. The higher incidence of EOD may result from limited awareness of prevention, non-standard GBS screening and IAP. When asymptomatic neonates were admitted to the NICU for prematurity, jaundice, maternal diabetes or chorioamnionitis, they received a diagnostic evaluation (blood cell count and blood/throat swab culture).This resulted in early detection, early diagnosis and early treatment.

Wortham reported that $13 \%$ of early onset infections had no documented symptoms, including 9\% who remained asymptomatic at $72 \mathrm{~h}$ but not refer to LOD [13]. Similarly, $11(11 / 67985,0.016 \%)$ infants with GBS-positive throat swab cultures were clinically healthy and received no antibiotics, which maybe due to infants' community and the prevalence of low pathogenic strains. Capsular polysaccharides (CPS) are thought to be associated with virulence of strains. CPS type III remains highly preponderant in cases of EOD and LOD, and $\mathrm{CC} 17$ possesses specific virulence traits that enhance its invasiveness [14-16]. Serotype III was the most prevalent serotype, followed by Ia, V, Ib and II. Neither serotype VII nor IX have been detected in China though it is expected that these two serotypes are associated with GBS [17]. We plan to identify the prevalent local serotype in the future.

The identification of risk factors remains a priority for prevention. Another important finding for this study is personal risk factors of EOD. In addition to previously acknowledged risk factors, we found neonatal GBS infections to be associated with membrane stripping and non-resident mother. Membrane stripping allows colonized GBS to ascend from the vagina to the amniotic fluid, and thus foetuses are exposed to GBS and aspirate the bacteria into their lungs $[6,18-21]$. Especially when membrane stripping fails to result in delivery, we suggest that providers consider the use of antibiotic prophylaxis. However, when penicillin, ampicillin, cefazolin or vancomycin is administered before delivery, the dose and duration must be precise according to a specific guideline or management protocol [6]. Guidelines such as the American College of Obstetrics and Gynecologists state that the optimal timing is $4 \mathrm{~h}$ prior to delivery.

As a retrospective study at a single hospital, our surveillance data indicates that when a caesarean delivery is performed after membrane stripping, the risk for EOD is similar to that of a vaginal delivery. The risk for transmission is likely much higher than in caesarean delivery before rupture of membranes or onset of labour $[8,22]$. Due to the vast population and high turnover, non-resident pregnant women who are low-income and without medical insurance may have poor living conditions and poor health care, which can lead to labour without prophylaxis. It is necessary to provide better management and proper medical care for non-resident women.

The overall mortality rate for neonatal GBS infections remains at approximately 10\% [2]. The case fatality rate of invasive GBS disease of $8.11 \%$ is similar to published reports. In a Japanese study, the case fatality rate of EOD cases was $13.6 \%$, which was much higher than that of LOD cases (8.0\%) [23]. Recent global data show the mean rates in cases of EOD and LOD to be $12.1 \%$ (95\% CI: 6.2-18.3) and 6.8\%(95\% CI: 4.3-9.4), respectively [2]. Prompt diagnostic evaluation will likely contribute to a lower fatality rate of EOD cases, and higher mortality of LOD cases may be due to delayed medical treatment. In contrast with Joubrel, who found lower gestational age and low birth weight to be risk factors for poor outcomes, no important predictors were found in our study [14]. Limitations of our study include GBS screened positive was not come up to the expectations. False negative results are expected because swabbing only the lower vagina decreases the culture yield substantially, and direct agar plating is used instead of selective enrichment broth [6]. This retrospective, single institution 
study has incomplete ascertainment of various variables that brought it limitations. The small sample size of cohort may affect the retrospective power. Fatality-related risk factors need further study due to the low prevalence of fatal cases.

\section{Conclusion}

In conclusion, our six-year investigation identified the incidence of invasive GBS infection in the Chinese mainland. This study demonstrates remarkable country-specific variation in comparison with other countries. Many individual risk factors were discovered for acquisition of invasive group B streptococcus disease. However, our findings can improve the awareness of neonatal GBS infection and lay a cornerstone to ensure accurate representation of the burden. With China's new two-child policy, a nationwide prevention strategy to reduce morbidity and mortality caused by GBS is urgently needed.

\section{Abbreviations}

EOD: Early onset disease; GBS: Group B streptococcus; LOD: Late onset disease; NICU: Neonatal intensive care unit

\section{Acknowledgements}

The authors sincerely thank all members who took part in this study.

\section{Funding}

Not applicable.

\section{Availability of data and materials}

The datasets supporting the conclusions of this article are included within the article.

\section{Authors' contributions}

QHY and JFD conceived and designed the study. QHY, XML and JLD performed the bacteriological analyses. QHY, XML and JLD collected the data. QHY, STW analyzed the data. QHY and STW wrote the manuscript. All authors reviewed and approved the final manuscript.

\section{Ethics approval and consent to participate}

This study was approved by the Ethics Committee of Shaoxing Women and Children's Hospital (NO 201700189). Because of retrospective studies, we contact babies' parents after discharge by telephone. Informed verbal consent was obtained from all participants and the ethics committee approved this procedure.

\section{Consent for publication}

Not applicable.

\section{Competing interests}

The authors declare that they have no competing interests.

\section{Publisher's Note}

Springer Nature remains neutral with regard to jurisdictional claims in published maps and institutional affiliations.

\section{Author details}

'Department of Clinical Laboratory, Shaoxing Women and Children's Hospital, 305 East Street Road, Shaoxing 312000, Zhejiang, China. ${ }^{2}$ University of International Business and Economics, 10 Huixing East Road, Beijing 100029, China.
Received: 23 August 2017 Accepted: 26 December 2018

Published online: 06 February 2019

\section{References}

1. Doare KL. Global maternal group B streptococcus colonisation. Lancet Infect Dis. 2016:16(9):992-3.

2. Edmond KM, Kortsalioudaki C, Scott S, Schrag SJ, Zaidi AK, Cousens S, et al. Group B streptococcal disease in infants aged younger than 3 months: systematic review and meta-analysis. Lancet. 2012;379(9815):547-56.

3. Fluegge K, Siedler A, Heinrich B, Schulte-Moenting J, Moennig MJ, Bartels $D B$, et al. Incidence and clinical presentation of invasive neonatal group B streptococcal infections in Germany. Pediatrics. 2006;117(6):e1139-45.

4. Heath PT, Balfour G, Weisner AM, Efstratiou A, Lamagni TL, Tighe $\mathrm{H}$, et al. Group B streptococcal disease in UK and Irish infants younger than 90 days. Lancet. 2004:363(9405):292-4.

5. Franciosi RA, Knostman JD, Zimmerman RA. Group B streptococcal neonatal and infant infections. J Pediatr. 1973;82(4):707-18.

6. Verani JR, McGee L, Schrag SJ. Prevention of perinatal group B streptococcal disease--revised guidelines from CDC, 2010. MMWR Recomm Rep. 2010; 59(RR-10):1-36.

7. Laboratory practices for prenatal Group. B streptococcal screening and reporting--Connecticut, Georgia, and Minnesota, 1997-1998. MMWR Morb Mortal Wkly Rep. 1999;48(20):426-8.

8. Schrag S, Gorwitz R, Fultz-Butts K, Schuchat A. Prevention of perinatal group B streptococcal disease. Revised guidelines from CDC. MMWR Recomm Rep. 2002:51(RR-11):1-22.

9. Deng JH, Yao KH, Hu HL, Yu SJ, Gao W, Fu LB, et al. Detection of group B streptococcus in the cases died of neonatal pneumonia. Zhonghua Er Ke Za Zhi. 2006:44(11):850-4

10. Yu HW, Lin HC, Yang PH, Hsu CH, Hsieh WS, Tsao LY, et al. Group B streptococcal infection in Taiwan: maternal colonization and neonatal infection. Pediatr Neonatol. 2011;52(4):190-5.

11. Zhang J, Zhao R, Dong Y, Zheng Y. Invasive group B streptococcal infection in infants in Shenzhen. China Int J Clin Exp Med. 2015;8(2):2939-43.

12. Schrag SJ, Zell ER, Lynfield R, Roome A, Arnold KE, Craig AS, et al. A population-based comparison of strategies to prevent early-onset group $B$ streptococcal disease in neonates. N Engl J Med. 2002;347(4):233-9.

13. Wortham JM, Hansen NI, Schrag SJ, Hale E, Van Meurs K, Sanchez PJ, et al. Chorioamnionitis and culture-confirmed, early-onset neonatal infections. Pediatrics. 2016;137(1):1-11.

14. Joubrel C, Tazi A, Six A, Dmytruk N, Touak G, Bidet P, et al. Group B streptococcus neonatal invasive infections, France 2007-2012. Clin Microbiol Infect. 2015:21(10):910-6.

15. Hong JS, Choi CW, Park KU, Kim SN, Lee HJ, Lee HR, et al. Genital group B Streptococcus carrier rate and serotype distribution in Korean pregnant women: implications for group B streptococcal disease in Korean neonates. J Perinat Med. 2010;38(4):373-7.

16. Johri AK, Paoletti LC, Glaser P, Dua M, Sharma PK, Grandi G, et al. Group B Streptococcus: global incidence and vaccine development. Nat Rev Microbiol. 2006:4(12):932-42.

17. Lu B, Li D, Cui Y, Sui W, Huang L, Lu X. Epidemiology of group B streptococcus isolated from pregnant women in Beijing, China. Clin Microbiol Infect. 2014;20(6):O370-3.

18. Yan JJ, Gong M, Zhang J, Zhu CB. The relationship between group B streptococcus genital infection and premature rupture of membrane. Zhonghua Yi Xue Za Zhi. 2016;96(23):1847-9.

19. Regan JA, Klebanoff MA, Nugent RP. The epidemiology of group B streptococcal colonization in pregnancy. Vaginal infections and prematurity study group. Obstet Gynecol. 1991:77(4):604-10.

20. Heinemann J, Gillen G, Sanchez-Ramos L, Kaunitz AM. Do mechanical methods of cervical ripening increase infectious morbidity? A systematic review. Am J Obstet Gynecol. 2008;199(2):177-87 discussion 87-8.

21. Hill MJ, McWilliams GD, Garcia-Sur D, Chen B, Munroe M, Hoeldtke NJ. The effect of membrane sweeping on prelabor rupture of membranes: a randomized controlled trial. Obstet Gynecol. 2008;111(6):1313-9.

22. Lopez Sastre JB, Fernandez Colomer B, Coto Cotallo GD, Ramos Aparicio A. Trends in the epidemiology of neonatal sepsis of vertical transmission in the era of group B streptococcal prevention. Acta Paediatr. 2005:94(4):451-7.

23. Matsubara K, Hoshina K, Suzuki Y. Early-onset and late-onset group B streptococcal disease in Japan: a nationwide surveillance study,2014-2010. Int J Infect Dis. 2013;17(6):e379-84. 